\title{
Tissue-specificity of 412 retrotransposon expression in Drosophila simulans and D. melanogaster
}

\author{
N Borie ${ }^{1}, C$ Maisonhaute ${ }^{2}$, S Sarrazin ${ }^{3}$, C Loevenbruck $^{1}$ and C Biémont ${ }^{1}$ \\ ${ }^{1}$ UMR CNRS 5558 'Biométrie et Biologie Evolutive', Université Lyon1 69622 Villeurbanne, France; ${ }^{2}$ UPR CNRS 9034 \\ 'Populations, Génétique et Evolution', 91198 Gif sur Yvette, France; ${ }^{3}$ Centre de Génétique Moléculaire et Cellulaire, Université \\ Lyon1 69622 Villeurbanne, France
}

\begin{abstract}
We analyse the expression of the retrotransposon 412 in the soma, testes, and ovaries in populations of Drosophila simulans and $D$. melanogaster, using RT-PCR and in situ hybridization. We find that expression of 412 is highly variable in the soma, confirming previous findings based on Northern blots. No 412 RNA is detected in the ovaries by either in situ hybridization or RT-PCR, in any population of either species. Transcripts are, however, detected in the male germline, which show a very characteristic spatial pat-
\end{abstract}

tern of 412 expression in primary spermatocytes. There is no relationship between expression of the 412 element in the soma and in the testes in the populations. These findings show that the expression of 412 is independently regulated in the soma and the testes, and this raises the question of the real influence of the somatic transcripts on the organism and on the transposition rate.

Heredity (2002) 89, 247-252. doi:10.1038/sj.hdy.6800135

Keywords: transposable element; transcription; germline; RT-PCR; in situ hybridization

\section{Introduction}

Retrotransposons are transposable elements (TEs) that transpose via an RNA intermediate. Transcription is a key step in the regulation of their transposition, the RNA being used as template for cDNA synthesis or as a messenger for translation into the proteins required for transposition. Retrotransposon expression thus depends on both cis-acting regulatory sequences from the elements themselves and trans-acting sequences from the host genome (Arkhipova et al, 1995; Matyunina et al, 1996). Interactions between these regulatory pathways lead to spatial and temporal patterns of expression.

TE expression patterns during embryogenesis (Ding and Lipshitz, 1994) and during the development of Drosophila (Parkhurst and Corces, 1987) were found to be identical for several TEs, which suggests that cis-regulatory sequences are conserved. In Drosophila, the expression of $I$, gypsy, and $P$ elements is greater in females than in males (Busseau et al, 1994; Pélisson et al, 1994; Roche et al, 1995), whereas the expression of micropia, 1731 and copia is mainly detected in males (Lankenau et al, 1994; Haoudi et al, 1997; Pasyukova et al, 1997). Such differences in TE expression may depend on the internal sequence of the element. Indeed, the expression of the $I$ factor in ovaries depends on an internal sequence of region 41-186 of the element (Udomkit et al, 1996), and the spatial expression patterns of the Fex element observed in embryos and in male and

Correspondence: C Biémont, UMR CNRS 5558, Laboratoire de Biométrie et Biologie Evolutive, Université Lyon1, 69622 Villeurbanne Cedex, France.E-mail: biemont@biomserv.univ-lyon1.fr

Received 29 May 2002; accepted 20 June 2002 female germlines are due to cis-regulatory sequences in region 295-762 (Kerber et al, 1996). Spatial and temporal TE expression patterns may also be influenced by host factors, such as developmental genes (Brookman et al, 1992; Frömmer et al, 1994), modifiers (Birchler and Hiebert, 1989; Rabinow et al, 1993; Birchler et al, 1994; Csink et al, 1994a,b), and hormones (Becker et al, 1991). For example, the embryonic expression of the 412 element results from interactions with the Ultrabithorax, $A b d A-B$ and pox meso developmental genes (Brookman et al, 1992; Ding and Lipshitz, 1994).

There have been very few population studies of TE expression. However, it has been shown that the copia transcript level differs in natural populations of $D$. melanogaster (Csink and McDonald, 1990), and that the amount of 412 transcripts during Drosophila development and in adults differs in different $D$. simulans populations according to the 412 copy number, whereas little or no expression is detected in D. melanogaster adults (Borie et al, 2000). No male/female differences in the amount of 412 transcripts have been detected for the whole-body in $D$. simulans, although sex-related differences may exist for 412 expression in germlines, as previously reported for the copia retrotransposon (Pasyukova et al, 1997). We analysed the spatial expression pattern of the 412 retrotransposon by in situ hybridization in testes and ovaries in several populations of D. simulans and D. melanogaster, and competitive RTPCR was used to compare RNA levels in carcasses, ovaries, and testes. 


\section{Methods}

\section{Natural populations}

We analysed 11 natural populations of $D$. simulans and three populations of $D$. melanogaster of various geographic origins, chosen according to their 412 copy number as determined by in situ hybridization (Vieira and Biémont, 1996). The populations of $D$. simulans were from Australia: Canberra (65 copies), Eden (15 copies), and Cann River (five copies); Russia: Moscow (20 copies); Portugal: Madeira (15 copies); Kenya: Kwale (four copies); New Caledonia: Noumea (five copies) and Amieu (seven copies); Madagascar (five copies); and Polynesia: Papeete $(20$ copies). The populations of $D$. melanogaster were from France: Valence (30 copies) and St Cyprien (30 copies), and China: Canton (18 copies). Flies of both species were reared in the laboratory at $18^{\circ} \mathrm{C}$.

\section{Transcript localization on whole mount ovaries and testes}

In situ experiments were done according to Tautz and Pfeifle (1989). Briefly, ovaries and testes from 5-day-old flies were dissected in $1 \times$ PBS, fixed with $4 \%$ paraformaldehyde, treated with proteinase $\mathrm{K}$ and simultaneously hybridized with an internal $1.8 \mathrm{~kb} 412$ labelled fragment. The fragment was obtained by PCR amplification of region 4283-6088 according to the complete 412 sequence described by Yuki et al (1986), and was labelled with the digoxygenin DNA labelling kit (Boehringer Mannheim/Roche), according to the manufacturer's instructions. Ten males and 10 females were analysed for each population.

\section{Competitive RT-PCR}

To compare 412 expression between somatic and germ lines, and between testes and ovaries, we performed competitive RT-PCR (Sigma Kit) on RNA extracted from carcasses (20-50 individuals), testes (50 individuals) and ovaries (20 individuals) as previously described (Borie et $a l, 1999)$. Duplicate extractions of these three tissues were performed for each population. An RNA competitor was made by in vitro transcription (AmpliScribe T7 Transcription kit, Epicentre Technologies). This was homologous with the 412 PCR fragment used in the in situ hybridization, apart from a deletion of the first 400 bases (cloned in pCRII using TA cloning system, Invitrogen), leading to an amplified fragment of $1.4 \mathrm{~kb}$ (Figure 1). This competitor could be amplified using the same set of oligonucleotides as the 412 element. The general principle used for producing such a homologous competitor is described in Schneeberger and Zeillinger (1995). We have adapted the method to generate a deletion instead of an insertion. We amplified the competitor fragment using as the forward primer a 45-mer (5'-TTCCTTTATATGGATGAC TTAATAG-CAGTACCCAGACTTCAGCAA-3') containing at its $3^{\prime}$ end a sequence homologous to region 4697-4716 (underlined) of the 412 element, and at its 5' end, the $5^{\prime}$ oligonucleotide used for 412 amplification (position 4283-4308, Yuki et al (1986)). The 3' oligonucleotide corresponded to the integrase region, position 60886063 (5'-TTCTATGACTTCTTTCTACTACTCC-3') of the 412 sequence (Yuki et al, 1986).

Before each RT-PCR experiment, RNA extracts were tested for DNA contamination and if appropriate treated with RNase free DNaseI. Previous experiments (data not shown) had shown that ideally the RT and PCR steps should be performed independently. RT reactions were carried out using $1 \mathrm{U} / \mu \mathrm{l} \mathrm{RT}, 1 \mu \mathrm{g}$ total RNA, and $2 \mathrm{pg}$ of the competitor with the $3^{\prime}$ oligonucleotide used for $\mathrm{PCR}$, at $42^{\circ} \mathrm{C}$ according to manufacturer's instructions. PCR $(0.05 \mathrm{U} / \mu \mathrm{l}$ taq polymerase) was performed on $7 \mu \mathrm{l}$ RT reaction for 30 runs (denaturing: $94^{\circ} \mathrm{C}$ for $45 \mathrm{sec}$; annealing: $50^{\circ} \mathrm{C}$ for $45 \mathrm{sec}$; elongation: $72^{\circ} \mathrm{C}$ for $2 \mathrm{~min}$ ). Competitive RT-PCR amplified two fragments, a $1.8 \mathrm{~kb}$ fragment corresponding to the 412 RNA transcript, and a $1.4 \mathrm{~kb}$ fragment corresponding to the competitor. For each tissue, two independent RNA extractions were done and used in RT-PCR experiments.

\section{Quantitative analysis}

Products of the RT-PCR amplifications were loaded on $1 \%$ agarose gels and transferred onto nylon membranes. Filters were hybridized with two different probes. This ultimate step increased the sensitivity of the method and ensured good specificity, since the probes did not hybridize with the primers. Probe 1 (Figure 1) corresponded to an internal $850 \mathrm{bp} M l u$-NI fragment of the 412 element located in the middle of the amplified region, and was used to detect both 412 and the competitor. Quantification was performed on the two expected fragments as described previously (Borie et al, 2000). Probe 2 (Figure 1) was 249 bp long and was generated by PCR between positions 71-319 of the 412 amplified fragment. This probe did not detect the competitor.

\section{Results}

\section{In situ localization of the 412 RNA transcript}

No 412 transcript was detected in the ovaries of the 14 populations analysed (Figure 2a). Conversely, whole mount testes showed a conserved 412 transcript pattern, with hybridization to the sub-terminal region of the testes (Figure $2 \mathrm{~b}-\mathrm{d}$ ), which corresponds to primary spermatocytes (Figure 2d). No signal was observed either in the apex, which corresponds to spermatogonia, or in the region of the testes where spermatocytes I develop into spermatocytes II and spermatozoa. This expression pattern was observed in all the populations of both $D$. simulans (Figure 2b) and D. melanogaster (Figure 2c), without any detectable differences in signal intensity between populations or between species.

\section{RNA amounts in germline extracts}

In agreement with results obtained by in situ hybridization, RT-PCR amplification of ovaries from the 14 populations showed no amplification of 412 RNA, although twice as much RNA was used for the ovaries as for the testes (data not shown). Since RT-PCR followed by radioactive detection of bands is a very sensitive method, these data suggest that 412 expression was strongly repressed in the ovaries. Even the Canberra population, which is known to have a very high expression of the 412 retrotransposon in whole females (Borie et al, 2000), did not exhibit any detectable 412 transcript in the ovaries.

412 transcripts were detected in the testes of all populations (Figure $3 \mathrm{a}$ ) of both D. simulans and D. melanogaster. Both the 412 and the competitor RNAs were detected. Quantification of these two fragments (Table 1) revealed 


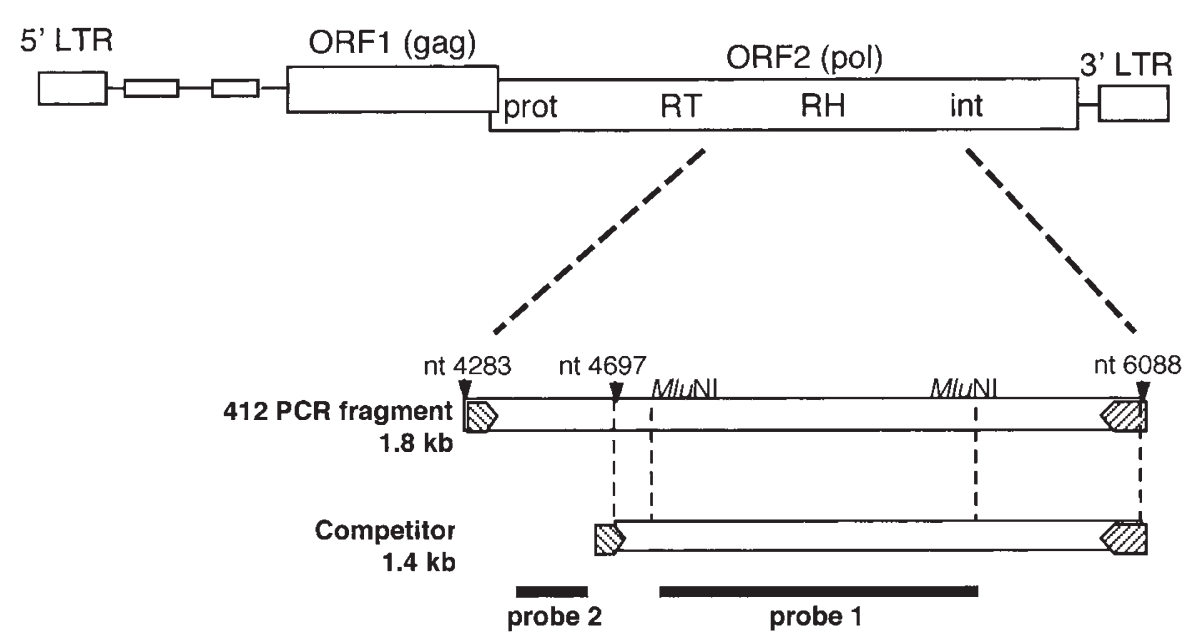

Figure 1412 fragments generated by RT-PCR. The $1.8 \mathrm{~kb}$ fragment is derived from the 412 RNA present in the sample. The $1.4 \mathrm{~kb}$ fragment corresponds to the amplification of the competitor with the same set of primers. Probes 1 and 2 used for further hybridization are shown at the bottom of the figure.
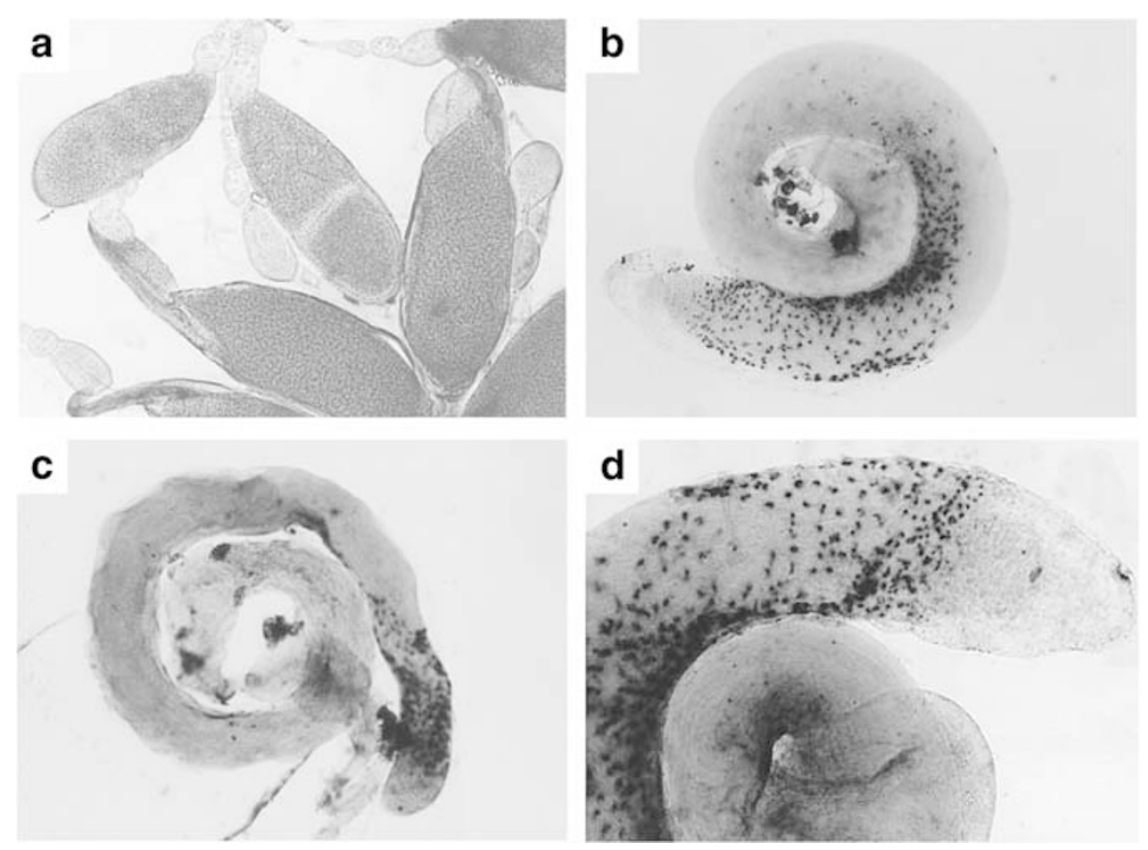

Figure 2 In situ localization of 412 RNA in ovaries from Drosophila simulans (a) and testes from populations of D. simulans (b) and D. melanogaster (c). In testes, the signal is detected only in primary spermatocytes (d).

that, in all these populations, the average 412 RNA amount corresponded to $2.16( \pm 1.08)$ times that of the competitor. In a second set of experiments we eliminated the competitor, making it possible to amplify potential RNAs expressed with an intensity lower than that of the $1.8 \mathrm{~kb}$ fragment. In addition to the expected $1.8 \mathrm{~kb}$ band, this experiment did indeed reveal a second band (Figure $4 \mathrm{a})$, of about $1.5 \mathrm{~kb}$, in the testes of all the populations (data not shown). Hybridization with probe 2 (Figure $4 \mathrm{~b}$ ) revealed two fragments, the $1.8 \mathrm{~kb}$ detected previously, and a second one of about $1.6 \mathrm{~kb}$ which was not detected by probe 1 , suggesting a deletion at the $3^{\prime}$ end of the amplified region. Probe 2 did not hybridize with the 1.5 $\mathrm{kb}$ fragment, suggesting that there could be a deletion in this region.
To check whether the $1.8 \mathrm{~kb}$ band corresponded to the full length 412 messenger RNA, we used two different $5^{\prime}$ primers located in the $5^{\prime}$ LTR. One was located just before the transcription start site (shown as - in Figure 5), and the other just after this site (indicated by + in Figure 5). The 3' primer was located $500 \mathrm{bp}$ downstream from the transcription start site. As seen in Figure 5, we failed to amplify any 412 fragment with primer (-), which suggests that the 412 messenger RNA detected began within the 412 promoter, and was not the result of host transcription read-through. Furthermore, the RT reactions were carried out with oligodT and with the $3^{\prime}$ primer located in the 5' LTR. The expected 500-bp fragment was detected with the two $5^{\prime}$ primers (Figure 5), suggesting that the 412 messenger RNAs were full length. 


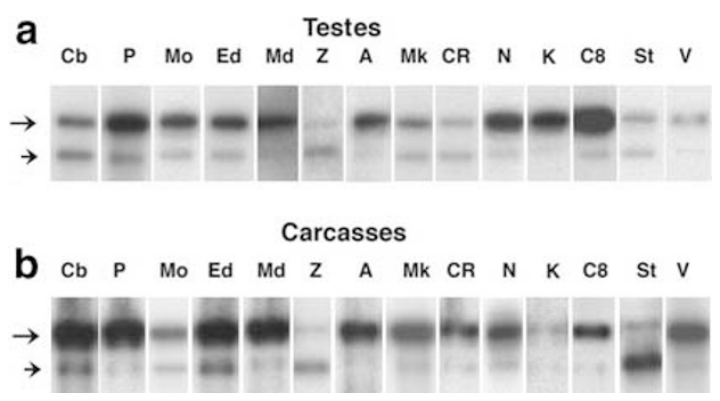

Figure 3 Competitive RT-PCR experiments on testes (a) and carcasses (b) from populations of Drosophila simulans: Canberra $(\mathrm{Cb})$, Papeete $(\mathrm{P})$, Moscow (Mo), Eden (Ed), Madeira (Md), Zimbabwe (Z), Amieu (A), Madagascar (Mk), Cann River (CR), Noumea (N), Kwale (K) and populations of D. melanogaster: Canton8 (C8), St Cyprien (St) and Valence (V). The long arrow indicates the $1.8 \mathrm{~kb}$ fragment corresponding to $412 \mathrm{RNA}$, and the short arrow indicates the $1.4 \mathrm{~kb}$ fragment originating from the competitor. The two fragments were revealed using probe 2, as described in Figure 1. Two picograms of competitor were added to all the samples, except in the carcasses from Canberra, Madeira, Eden and St Cyprien, where the competitor amount added was between 10 and $40 \mathrm{pg}$.

Table 1 Intensity of the $1.8 \mathrm{~kb}$ band of the 412 element in 14 populations of Drosophila simulans and D. melanogaster, in carcasses and in testes

\begin{tabular}{llrrr}
\hline \multirow{2}{*}{ Species } & Population & \multirow{2}{*}{412 copy no. } & \multirow{2}{*}{412 RNA amount } \\
\cline { 3 - 5 } & & & Soma & Testes \\
\hline & Canberra & 65 & 39.7 & 1.3 \\
& Papeete & 20 & 2.4 & 2.4 \\
& Moscow & 20 & 2.3 & 3.3 \\
& Eden & 15 & 17.9 & 3.0 \\
& Madeira & 15 & 20.9 & 3.7 \\
D. simulans & Zimbabwe & 9 & 0.2 & 0.5 \\
& Amieu & 7 & 3.9 & 4.2 \\
& Noumea & 6 & 3.5 & 3.6 \\
& Cann River & 5 & 2.9 & 1.4 \\
D. melanogaster & Madagascar & 5 & 10.6 & 2.3 \\
& St Cyprien & 30 & 2.0 & 1.1 \\
& Valence & 30 & 8.8 & 1.1 \\
Mean & Canton & 18 & 3.6 & 2.2 \\
SD & & & & \\
& & & 11.2 & 2.3 \\
\hline
\end{tabular}

The values are relative to the competitor. SD: standard deviation.

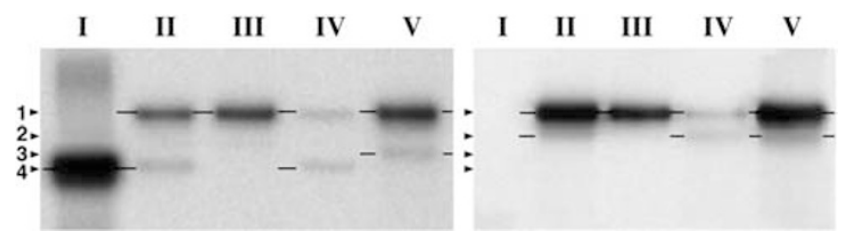

Figure 4 Competitive RT-PCR controls. Competitor alone (I), carcasses with competitor (II), carcasses without competitor (III), testes with competitor (IV), testes without competitor (V). The same blot was hybridized with two different probes. (a) Hybridization with probe 1 , which reveals the $1.8 \mathrm{~kb}$ fragment (1), the $1.4 \mathrm{~kb}$ fragment corresponding to the competitor (4), and a third $1.5 \mathrm{~kb}$ fragment found in testes (3). (b) Hybridization with probe 2, which does not reveal the competitor, but does reveal a $1.6 \mathrm{~kb}$ fragment (2) instead.

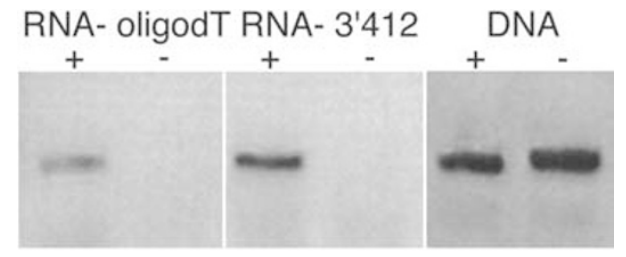

Figure 5 PCR controls. The cDNA generated either from oligodT or 412 specific primer located in the $5^{\prime}$ LTR was used in the PCR with one $5^{\prime}$ primer located downstream $(+)$ or upstream (-) from the transcription start site. A PCR of DNA extracts was also used as a positive control.

\section{RNA amount in soma extracts}

Because the 412 element is expressed at the same level in whole males and females (Borie et al, 2000), we analysed 412 expression in pooled male and female carcasses (Figure 3b). Table 1 shows the estimated 412 RNA amount in soma and testes for all the populations tested. Band intensity was much higher for 412 RNA than for the competitor, with the average 412 amount 9.14 times $( \pm 10.75)$ that of the competitor. Since the competitor is present in equal amounts in the soma and testes, this suggests that 412 element was expressed to a greater exent in soma than in testes. The amount of 412 RNA appears to be more variable in carcasses than in testes among populations. In D. simulans, a very high level of 412 expression was observed in the populations from Canberra (40-fold higher than that of the competitor), Madeira (21-fold) and Eden (18-fold), and the lowest level of expression was detected in the African population from Zimbabwe (0.2 times the competitor amount). This very low expression level for 412 element in Zimbabwe is consistent with the absence of the 412 transcript in previous Northern blot experiments (Borie et al, 2000). In D. melanogaster, the somatic expression of 412 was higher in the population from Valence than in Canton or St Cyprien. Experiments done without the RNA competitor (Figure $4 \mathrm{a}$ ) did not detect in carcasses the $1.5 \mathrm{~kb}$ fragment observed in testes, suggesting that this fragment may be testis-specific. The $1.6 \mathrm{~kb}$ fragment detected with probe 2 in the testes was also detected in the soma (Figure $4 \mathrm{~b}$ ), and so cannot be tissue-specific. Since probe 2 is short and several transposable elements are phylogenetically close to the 412 element, we cannot rule out the possibility that this fragment results from the amplification of one of the other transposable elements.

Statistical analysis of the 412 RNA amounts from testes and soma reveals the absence of correlation between expression in testes and in soma $\left(r^{2}=0.00004, P=0.98\right)$, suggesting that 412 expression is regulated independently in these two tissues.

\section{Discussion}

412 is expressed only in males

Various studies have shown that the expression of transposable elements is regulated differently according to the sex of the fly. $P$ transposon transcription is regulated in such a way that transposition is allowed only in the germline, due to alternative splicing of complete messenger RNA (Roche et al, 1995); the I factor is expressed mainly in the females' ovaries, allowing transposition in female germinal cell line (Busseau et al, 1994). However, 
the regulation of the transcription and transposition of these two elements has been reported as a result of crosses under conditions implying hybrid dysgenesis. In non-dysgenic crosses, the activity of the $I$ and $P$ elements is totally or partially repressed (Busseau et al, 1994; Coen et al, 1994). Expression of the transposable elements therefore seems to be severely controlled in germlines, presumably to limit any increase in the copy number with its associated deleterious effects. This is the case for the copia retrotransposon, which is very weakly expressed in testes from stable $D$. melanogaster strains, but strongly expressed in the same tissue from unstable strains (Pasyukova et al, 1997). We show here that expression of the 412 retrotransposon is different in males and females of both $D$. simulans and $D$. melanogaster. There was no detectable expression of the 412 retrotransposon in the ovaries, but it was expressed in the testes, with a highly conserved spatial pattern, suggesting that 412 expression in males is regulated by internal sequences. The fact that 412 is only expressed in the testes of the males implies that 412 transposition may only occur in the male germline, as observed for the copia retrotransposon (Pasyukova et al, 1997). The restriction of this transposition to the male germline should lead to there being more 412 insertions on the $\mathrm{Y}$ chromosome, and fewer on the $X$ chromosome than on the autosomes, because the male is hemizygous in Drosophila. This is consistent with the observation that both $D$. simulans and D. melanogaster show numerous insertions of 412 and copia retrotransposons on the $Y$ chromosome (Junakovic et al, 1997) with many times fewer insertions on the $X$ chromosome than on the autosomes (Biémont, 1992; Biémont et al, 1997).

\section{A testis-specific transcript}

In addition to the expected $1.8 \mathrm{~kb}$ fragment, the RT-PCR analysis of testis extracts has revealed a second fragment of about $1.5 \mathrm{~kb}$, present at low levels. This suggests the presence of a second messenger RNA that is specific to the testes. This RNA could result from an internal deletion in the integrase-RT region of the 412 sequence, since it was amplified by the same set of primers as the $1.8 \mathrm{~kb}$ fragment. This possible deletion would have to occur within the first $300 \mathrm{pb}$, since the fragment was not detected using probe 2. This new messenger RNA may also be derived from a small messenger produced from complete copies of 412 by splicing events.

\section{Somatic and germinal expressions are independently regulated}

Although both somatic and germinal expressions of the 412 retrotransposon appear to be regulated, the regulation mechanisms involved seem to be different. In the soma, 412 expression was highly variable in different populations and varied with developmental stage. This expression of 412 appears to be associated in some way with the copy number, at least in D. simulans (Borie et al, 2000). The picture is more complex in D. melanogaster where somatic transcription was detected in RT-PCR experiments, whereas no transcript had been revealed by previous Northern blot analyses done on total mRNA from whole organisms (Borie et al, 2000). This discrepancy may result either from technical differences, or from changes in the characteristics of the populations over time, as has previously been reported for the Moscow population (Borie et al, 2000).
The relatively constant amount of 412 transcript in the male germline in all the populations, contrasted sharply with the somatic differences in the transcript amount, which varied between populations according to copy number (Borie et al, 2000). This suggests that precise regulation of 412 transcription occurs in the male germline, independently of the whole organism. Because the transposition rate of 412 has been found to be the same in various populations of D. simulans (Vieira and Biémont, 1997), the apparently constant transcription in the male germline suggests that transposition is regulated at the transcription level, and is independent of copy number. This is particularly evident in the Canberra population, which has a very high 412 copy number and a high level of somatic expression, but the same transposition rate in males (Vieira and Biémont, 1996) and the same germinal transcription rate as the other populations. Transcriptional regulation of transposition in germline has previously been reported for the copia retrotransposon in unstable lines of $D$. melanogaster (Pasyukova et al, 1997; Filatov et al, 1998), but in these lines, a high transcription rate was found to be associated with a high transposition rate. No somatic transposition was observed for copia, even though this element had a high somatic transcription rate, which was positively correlated with copy number (Pasyukova et al, 1997; Nuzhdin et al, 1998). As a result, the regulation of transposition at the translational level in the soma, as found for the 1731 element in germline (Brierley and Flavell, 1990; Haoudi et al, 1997), should be of more interest.

One important question is what makes transposable elements transcribe differently in the whole organism and in the germline. In some cases, only a few copies may be expressed in the germline as compared to the soma, whereas in others, the expressed copies may differ in sequence, perhaps as a result of the presence of specific internal cis-regulatory sequences. Indeed, the expression of a testis-specific antisense RNA in the micropia element has been shown to depend on the presence of a specific promoter within the LTR (Lankenau et al, 1994). Kalmykova et al (1999) recently showed that expression of the 1731 retrotransposon in testes was due to a specific copy. Even if the regulation of transcription in germlines has evolved as a reaction against TE transposition, the large amount of transcripts produced in the soma during development is a puzzling waste of energy for the cells and the organisms. This over-expression of TE RNA may be involved in various cellular processes. However, the decrease in the global expression of 412 with copy number in many populations does not fit in with such a global positive effect. Further experiments are called for to elucidate the regulation of somatic expression of the 412 element.

This work was supported by the Centre National de la Recherche Scientifique, the GDR 2157 on Transposable Elements, the Bureau des Ressources Génétiques, and the Association pour la Recherche sur le Cancer. We would like to thank Monika Ghosh for revising the English text.

\section{References}

Arkhipova IR, Lyubomirskaya NV, Ilyin YV (1995). Drosophila retrotransposons. Springer Verlag: Germany.

Becker J, Micard D, Becker JL, Fourcade-Peronnet F, Dastugue B, Best-Belpomme M (1991). Ecdysone decreases the transcrip- 
tion level of the retrotransposons 1731 and 412 in a Drosophila cell line. Cell Mol Biol 37: 41-49.

Biémont C (1992). Population genetics of transposable DNA elements. A Drosophila point of view. Genetica 86: 67-84.

Biémont C, Tsitrone A, Vieira C, Hoogland C (1997). Transposable element distribution in Drosophila. Genetics 147: 19971999.

Birchler JA, Hiebert JC (1989). Interaction of the Enhancer of white-apricot with transposable element alleles at the white locus in Drosophila melanogaster. Genetics 122: 129-138.

Birchler JA, Bhadra U, Rainbow L, Lins R, Nguyen-Huynh T (1994). Weakener of white (Wow), a gene that modifies the expression of the white eye color locus and that suppresses position effect variegation in Drosophila melanogaster. Genetics 137: 1057-1070.

Borie N, Loevenbruck C, Biémont C (1999). Developmental expression of the rp49 gene in Drosophila melanogaster and D. simulans. Dros Inf Serv 82: 78-79.

Borie N, Loevenbruck C, Biémont C (2000). Developmental expression of retrotransposon 412 in natural populations of D. melanogaster and D. simulans. Genet Res 76: 217- 226.

Brierley C, Flavell AJ (1990). The retrotransposon copia controls the relative levels of its gene products post-transcriptionally by differential expression from its two major mRNAs. Nucl Acids Res 18: 2947-2951.

Brookman JJ, Toosy AT, Shashidhara LS, White RAH (1992). The 412 retrotransposon and the development of gonadal mesoderm in Drosophila. Development 116: 1185-1192.

Busseau I, Chaboissier MC, Pélisson A, Bucheton A (1994). I factors in Drosophila melanogaster : transposition under control. Genetica 93: 101-116.

Coen D, Lemaitre B, Delattre M, Quesneville H, Ronsseray S, Simonelig M et al (1994). Drosophila $P$ element: transposition, regulation and evolution. Genetica 93: 61-78.

Csink AK, McDonald JF (1990). Copia expression is variable among natural populations of Drosophila melanogaster. Genetics 126: 375-385.

Csink AK, Linsk R, Birchler JA (1994a). The Lighten up (Lip) gene of Drosophila melanogaster, a modifier of retroelement expression, position effect variegation and white locus insertion alleles. Genetics 138: 153-163.

Csink AK, Linsk R, Birchler JA (1994b). Mosaic suppressor, a gene in Drosophila that modifies retrotransposon expression and interacts with zeste. Genetics 136: 573-583.

Ding D, Lipshitz HD (1994). Spatially regulated expression of retrovirus-like transposons during Drosophila melanogaster embryogenesis. Genet Res 64: 167-181.

Filatov DA, Nuzhdin SV, Pasyukova EG (1998). Enhanced transcription of the copia retrotransposon in Drosophila melanogaster testes. Mol Biol 32: 821-824.

Frommer G, Schuh R, Jäckle H (1994). Localized expression of a novel micropia-like element in the blastoderm of Drosophila melanogaster is dependent on the anterior morphogen bicoid. Chromosoma 103: 82-89.

Haoudi A, Rachidi M, Kim MH, Champion S, Best-Belpomme M, Maisonhaute C (1997). Developmental expression analysis of the 1731 retrotransposon reveals an enhancement of gagpol frameshifting in males of Drosophila melanogaster. Gene 196: 83-93.

Junakovic N, Terrinoni A, Di Franco C, Vieira C, Loevenbruck
C (1997). Accumulation of transposable elements in the heterochromatin and on the Y chromosome of Drosophila simulans and Drosophila melanogaster. J Mol Evol 46: 661-668.

Kalmykova A, Maisonhaute C, Gvozdev V (1999). Retrotransposon 1731 in Drosophila melanogaster changes retrovirus-like expression strategy in host genome. Genetica 107: 73-77.

Kerber B, Fellert S, Taubert H, Hoch M (1996). Germ line and embryonic expression of Fex, a member of the Drosophila Felement retrotransposon family, is mediated by an internal cis-regulatory control region. Mol Cell Biol 16: 2998-3007.

Lankenau S, Corces VG, Lankenau DH (1994). The Drosophila micropia retrotransposon encodes a testis-specific antisense RNA complementary to reverse transcriptase. Mol Cell Biol 14: 1764-1775.

Matyunina LV, Jordan IK, McDonald JF (1996). Naturally occuring variation in copia expression is due to both element (cis) and host (trans) regulatory variation. Proc Natl Acad Sci USA 96: 7097-7102.

Nuzhdin SV, Pasyukova EG, Morozova EA, Flavell AJ (1998). Quantitative genetic analysis of copia retrotransposon activity in inbred Drosophila melanogaster lines. Genetics 150: 755-766.

Parkhurst SM, Corces VG (1987). Developmental expression of Drosophila melanogaster retrovirus-like transposable elements. EMBO J 6: 419-424.

Pasyukova EG, Nuzhdin, SV, Li W, Flavell AJ (1997). Germ line transposition of the copia retrotransposon in Drosophila melanogaster is restricted to males by tissue-specific control of copia RNA levels. Mol Gen Genet 255: 115-124.

Pélisson A, Song SU, Prud'homme N, Smith P, Bucheton A (1994). Gypsy transposition correlates with the production of a retroviral envelop-like protein under the tissue-specific control of Drosophila flamenco gene. EMBO J 13: 4401-4411.

Rabinow L, Chiang Su L, Birchler JA (1993). Mutations at the darkener of apricot locus modulate transcript levels of copia and copia-induced mutations in Drosophila melanogaster. Genetics 134: 1175-1185.

Roche SE, Schiff M, Rio DC (1995). P element repressor autoregulation involves germ-line transcriptional repression and reduction of third intron splicing. Genes Dev 9: 1278-1288.

Schneeberger C, Zeillinger R (1995). PCR-mediated synthesis of exogenous competitors for quantitative RT-PCR. BioTechniques 20: $360-362$.

Tautz D, Pfeifle C (1989). A non-radioactive in situ hybridization method for the localization of specific RNAs in Drosophila embryos reveals translational control of the segmentation gene hunchback. Chromosoma 98: 81-85.

Udomkit A, Forbes S, Arkhipova I, McLean C, Finnegan DJ (1996). Control of expression of the I factor, a LINE-like transposable element in Drosophila melanogaster. EMBO J 15: 3174-3181.

Vieira C, Biémont C (1996). Geographical variation in insertion site number of retrotransposon 412 in Drosophila simulans. J Mol Evol 42: 443-451.

Vieira C, Biémont C (1997). Transposition rate of the 412 retrotransposable element is independent of copy number in natural populations of Drosophila simulans. Mol Biol Evol 14: 185-188.

Yuki S, Inouye S, Ishimaru S, Saigo K (1986). Nucleotide sequence characterization of a Drosophila retrotransposon, 412. Eur J Biochem 158: 403-410. 